\title{
INTEGRAÇÃO DOS OBJETOS TECNOLÓGICOS E SUA DIMENSÃO NORMATIVA AO INFERENCIALISMO PRAGMÁTICO
}

\author{
Ralph Leal Heck ${ }^{1}$
}

\begin{abstract}
Resumo
As questões que se colocam neste artigo são: em que sentido os objetos tecnológicos trazem intrinsecamente um caráter prático e normativo e como podemos concebê-los nestas dimensões, tomando-as em um caráter constitutivo? Desta forma, eles podem ser projetados para estender ou realizar ações no plano das práticas constitutivas do humano enquanto tal, que da perspectiva de Brandom, estas práticas constitutivas correspondem à dimensão linguístico-pragmática. Para realizar isto, inicio com a leitura pragmática dada por Bunge aos objetos tecnológicos. No tópico seguinte, discuto como Franssen compreende a dimensão normativa constitutiva dos objetos tecnológicos. A partir destas perspectivas, apresento o inferencialismo de Brandom e me aproprio das concepções de Bunge e Franssen lendo-as no interior do inferencialismo. Concluo, que dados certos ajustes epistêmicos na concepção de Bunge e ajustes ontológicos na concepção de Franssen, podemos tratar a definição das propriedades dos objetos a partir do seu design e da prática dos seus usuários. Uma definição que não é estática e poder sofrer intervenções dos usuários sobre o design e vice versa. Outro resultado é que podemos pensar estes objetos como proto-agentes e extensores de ações acopladas aos usuários, uma vez que, eles são tratados no interior do plano constitutivo próprio do humano com grande multiplicidade e detalhe fornecidos pela abordagem de Brandom.
\end{abstract}

Palavras-chave: Filosofia da Tecnologia. Pragmática. Normatividade. Inferencialismo.

\section{INTEGRATION OF TECHNOLOGICAL OBJECTS AND THEIR NORMATIVE DIMENSION TO PRAGMATIC INFERETIALISM}

\begin{abstract}
The questions that arise in this article are: in what sense do technological objects intrinsically bring a practical and normative character and how can we conceive them in these dimensions, taking them into a constitutive way? Through this they can be designed to extend or carry out actions in terms of human constitutive practices as such, which, from Brandom's perspective, these constitutive practices correspond to the linguistic-pragmatic dimension. To accomplish this, I begin with the pragmatic reading given by Bunge to technological objects. In the next topic, I discuss how Franssen understands the normative dimension that constitutes technological objects. From these perspectives, I present Brandom's inferentialism and take ownership of the concepts of Bunge and Franssen by reading them within inferentialism conceptual framework. I conclude that given certain epistemic adjustments in Bunge's conception and ontological adjustments in Franssen's conception, we can deal with the definition of the properties of objects based on their design and the practice of its users. A definition that is not static and can suffer interventions from users over the design and vice versa. Another result is that we can think of these objects as proto-agents and extenders of actions coupled to users, since they are treated within the constitutive plane of the human being in great multiplicity and detail provided by Brandom's approach.
\end{abstract}

Key-words: Philosophy of Technology. Pragmatics. Normativity. Inferentialism.

1 Possui graduação em Filosofia pela Universidade Estadual do Ceará (2008), mestrado em Filosofia pela Universidade Federal do Ceará (2012) e doutorado em Filosofia pela Universidade Federal do Ceará (2018). Atualmente é professor substituto da Universidade Federal do Ceará, professor de filosofia da Faculdade Católica de Fortaleza. Tem experiência na área de Filosofia, com ênfase em Filosofia Analítica, atuando principalmente nos seguintes temas: filosofia da linguagem, lógica, filosofia da informação, metafísica, filosofia da ciência e filosofia mente. E-mail: imagomundi@hotmail.com.

\begin{tabular}{|l|c|c|c|c|}
\hline Renista Dialectus & Ano 9 & n. 19 & Agosto-Dezembro 2020 & p. $162-175$ \\
\hline
\end{tabular}




\section{Introdução}

Atualmente, a tecnologia desempenha um papel sem precedentes na organização social e na própria tessitura das sociedades. Boa parte de nossas tomadas de decisão passam por artefatos tecnológicos, quando não, são de algum modo registradas ou computadas por eles. Esta percepção de amálgama entre nós e as máquinas, essa fusão onto-epistemológica, quase sem atrito com os artefatos, aceleram nossas ações e ampliam seu alcance. Fazendo com que os itens tecnológicos sejam tratados como parte de nós ou mesmo como entidades autônomas a quem atribuímos desejos, vontades e preferencias.

Nosso domínio teórico de partida é a filosofia da tecnologia. Ela é uma disciplina transversal às áreas clássicas da literatura filosófica como epistemologia, ontologia, estética, ética e política. Segundo Franssen (2018), há ao menos quatro raízes do pensamento filosófico sobre a tecnologia: a primeira raiz está na ideia de que nossa técnica e artefatos tecnológicos procuram imitar a natureza. A segunda raiz está voltada para a diferença ontológica entre artefatos naturais e artefatos tecnológicos, onde os artefatos são distintos em razão de sua capacidade intrínseca de mudança e geração. E, por causa disso, os artefatos tecnológicos necessitam de manutenção humana constante. A terceira raiz discute os princípios teleológicos dos artefatos tecnológicos, influenciados até hoje pelos quatro princípios causais de Aristóteles (material, formal, eficiente e final). A quarta raiz discute o papel que os artefatos tecnológicos desempenham como imagem para explicar e expandir a compreensão do funcionamento da realidade.

Nossa entrada neste tema será pela terceira raiz. Como todos os itens feitos por nós, os itens tecnológicos possuem um telos, um objetivo, uma meta para cumprir em seu uso inicialmente pensado, mas diferente de Aristóteles, tomarei este telos pelo prisma da filosofia de Mario Bunge sobre a diferença entre a ciência e tecnologia. E uma vez que tenhamos compreendido qual é o papel da tecnologia em nossa interação com a realidade e sua composição, farei uma incursão na ideia de que o próprio modo como nós entendemos os artefatos tecnológicos segue a estrutura de nossa racionalidade. Em outras palavras, o modo constitutivo da finalidade tecnológica é da mesma natureza de nossas práticas sociais, por isto, é interessante estudá-los de uma perspectiva normativa e, mais detalhadamente, a partir de uma perspectiva inferencial-pragmática.

\begin{tabular}{|l|c|c|c|c|}
\hline Qevista Dialectus & Ano 9 & n. 19 & Agosto - Dezembro 2020 & p. $162-175$ \\
\hline
\end{tabular}




\section{Aspectos práticos da tecnologia em Bunge}

Bunge compreende a tecnologia como ciência aplicada, isto é, o conhecimento científico empreendido com o objetivo de satisfazer algum tipo de interesse exterior à agenda da ciência. A ciência como conhecimento teórico ocupa-se com problemas no sentido de compreensão da realidade. Esta compreensão, por sua vez, nos fornece um cenário descrito pela ciência nos permitindo uma atuação planejada sobre a realidade com o objetivo de efetivar outros objetivos. Ações e metas demandadas (quer por governos, setores da sociedade, grupos de indivíduos, quer por indivíduos, etc.) encontram sua criação e resolução através do cenário teórico-científico.

Assim, dos nossos fazeres surgem dificuldades e limitações, que se pode tentar resolver via ciência aplicada. Segue-se daí a pesquisa científica, para então a ação racional. Um dos tipos de ação racional é a ação tecnológica, que é a ação de produzir artefatos para esta ou aquela finalidade. (BUNGE, 1966. p.330). Um exemplo claro disto é a quantidade de pesquisas desenvolvidas e medicamentos em desenvolvimento desde a descoberta da nova corona vírus e tantos outros medicamentos com ação antiviral que foram adaptados para tentar conter ou eliminar a covid-19.

A ação tecnológica tem em vista dois tipos de teoria: a substantiva e a operativa. A teoria substantiva emprega as teorias científicas diretamente sobre o mundo de modo a maximizar certos efeitos. Por exemplo, a aplicação da dinâmica de fluidos na fabricação de aviões para dar aerodinâmica a eles. Ou o estudo de nanopartículas na fabricação de condutores elétricos otimizados para a construção de computadores velozes e eficientes. Já as teorias operativas, estão voltadas para a operação humana e complexos homem-máquina em situações reais, a tecnologia empregada no conforto durante o transporte de um avião, ou o design intuitivo de um celular ou aplicativo. Isto que cria uma enorme diferença entre estas duas ações. De um lado, a aplicação substantiva do conhecimento sobre a mecânica gravitacional nos permite produzir foguetes que consomem menos energia para atingir o espaço ou menos combustível para se deslocar no sistema solar. Por outro, a aplicação operativa, que pode ser explicada pelo uso da psicologia cognitiva para construir imagens, desenhos, figuras, cores e formas mais agradáveis e convincentes a determinados tipos de pessoas em filmes e propagandas. Tal conhecimento assegura o sucesso do objetivo (fazer comprar um produto x ou y que em nada tem a ver com a psicologia cognitiva). Neste sentido, o conhecimento científico

\begin{tabular}{|l|c|c|c|c|}
\hline Qevista Dialectus & Ano 9 & n. 19 & Agosto-Dezembro 2020 & p. $162-175$ \\
\hline
\end{tabular}


amplifica o design de nossas ações que, por sua vez, são dotadas de finalidades completamente diversas ao domínio científico evocado para aprimorá-las.

Segundo Bunge, as teorias que fundamentam a ação operativa são teorias da decisão, teorias do valor, teoria dos jogos e etc. Elas visam aperfeiçoar uma resposta (um output) dado determinado input diante de quaisquer ferramentas que a ciência possa disponibilizar e materializar. De fato, Bunge afirma que as ciências formais e o conhecimento ordinário são suficientes para desenvolver algum conhecimento operativo. Mas, em que este conhecimento se assemelha à ciência então? A semelhança está no método. Elas são teorias científicas da ação. Essas teorias são tecnológicas no que diz respeito ao meio pelo qual a ação se desenrola. Já os objetivos da teoria são práticos e não cognitivos (ou epistêmicos) como são os da ciência. Sendo, portanto, teoricamente mais rasas.

As teorias que orientam a ação operativa dependem de 4 características: 1) Estas teorias se referem a modelos idealizados da realidade (usuários, e efeitos idealizados). 2) Tem aplicados conceitos teóricos (probabilidade, causalidade etc.); 3 ) é capaz de absorver informação para enriquecer a experiência do usuário ou o resultado da teoria, fornecendo regulações, predições, retrodições e 4) são empiricamente testáveis.

Apesar de rasas, tais teorias são mais ricas de aplicação por serem orientadas ao que deve acontecer ao manuseá-las, diferente do objetivo de prever e explicar eventos como é o caso do conhecimento científico. A ação tecnológica, por meio dos modelos, cria uma caixa preta, onde o que interessa é a entrada e a saída da caixa explicado pelos termos teóricos das teorias científicas normalmente usadas como componentes desta caixa.

As características (1) e (2) dão vazão às várias características e funcionalidades tecnológicas, dentre elas, o desenho do produto que se apresenta a nós usuários, como a sua própria condição de existência e teoricidade. Ambas completamente embebidas em uma agenda prática, proveniente das características (3) e (4): O controle, teste e ajuste de erros são feitos, obviamente, pela via do uso do equipamento, retroalimentando sua estrutura e fortalecendo sua capacidade e eficiência em alcançar a finalidade desenhada. As perguntas típicas que orientam estas estratégias são: "Isto funciona?", "Pode funcionar mais eficientemente?", "Pode aprender com os erros?", "Pode se ajustar aos usuários?"

\begin{tabular}{|l|c|c|c|c|}
\hline Qevista Dialectus & Ano 9 & n. 19 & Agosto - Dezembro 2020 & p. $162-175$ \\
\hline
\end{tabular}


E aqui nos distanciamos de Bunge. Pois, para ele, há uma separação epistêmica fundamental entre teoria e prática, entre know-how e know-that. Diferente dele assumo que o know-that é um tipo específico de know-how, onde o know-that nasce do know-how, tal que, este o amplifica. Além disso, não concordo com a separação que Bunge faz da atividade científica orientada por leis e a atividade tecnológica orientada por regras (Idem. p.338). No meu entendimento, as leis são regras mais rígidas e resistentes que as regras convencionais. Mas, não diferem essencialmente em estatuto. A rigidez e resistência das leis científicas ${ }^{2}$ se dão por seu entrelaçamento próprio com várias outras normas e práticas científicas, tornando sua revogação, modificação etc. mais difíceis.

\section{O estatuto normativo da tecnologia em Franssen}

Como estamos dedicados neste momento a investigar como a tecnologia amplifica nossas ações e nosso conhecimento, explorarei a direção do know-how para o know-that. Bunge afirma que o desenvolvimento tecnológico habilita o desenvolvimento da ciência em razão de novas perguntas que somos capazes de fazer. Como a tecnologia nos permite fazer mais coisas, ela nos permite fazer perguntas adicionais com menos esforços ou com a aplicação mais intensa da própria tecnologia.

Este duplo fazer, o fazer do objeto (artefato) e o fazer habilitado por ele, vinculam profundamente estes itens com a dimensão normativa de nossas vidas, ponderados a partir de nossos juízos sobre o fazer eficiente ou ineficiente, bom ou ruim, maléfico ou benéfico. Com efeito, segundo (FRANSSEN, 2006.), estas características são constitutivas do que distingue os artefatos tecnológicos dos artefatos naturais. Não apenas podemos falar de bons e maus artefatos, mas toda forma de atribuição funcional ${ }^{3}$ a um artefato é de caráter normativo. O normativo é caracterizado por ele em um amplo espectro, não redutível ao puramente físico, mas inerentemente composto por fatos intensionais (Idem. p.44), o que coloca fatos avaliativos como parte dos fatos normativos. Seguindo esta organização, há fatos relevantes nas nossas tomadas de decisão, desejos e crenças que dirigem a funcionalidade dos artefatos. A relevância é considerada por ele

2 O que levo aqui em consideração é o estatuto epistêmico de uma lei. Por mais que tenhamos muita confiança em uma lei, não há garantias metafísicas de que esta seja de fato a lei. (Hume, Russell e Popper já mostraram isto).

3 Atribuição funcional significa o que dizemos que ele é capaz e fazer e ao que ele foi projetado para fazer.

\begin{tabular}{|l|c|c|c|c|}
\hline Q Povista Dialectus & Ano 9 & n. 19 & Agosto-Dezembro 2020 & p. $162-175$ \\
\hline
\end{tabular}


em três níveis: teorético, prático e oréctico (mental) e há dois tipos de relevância: 1) um fato que leva certa pessoa a ter uma razão para fazer, acreditar, desejar algo e 2) um fato que leva uma pessoa a dever fazer, acreditar, desejar algo. Além disso, há a distinção entre a relevância fundada racionalmente e a relevância fundada na moral, onde nossas ações se encontram embebidas em ambas (Idem. p.45). A relevância racional lida com as razões para se utilizar (ou não utilizar) determinado artefato para determinada finalidade e a relevância moral diz respeito aos efeitos e consequências benéficos ou maléficos do uso do artefato, que normalmente se espera que sejam explicitados pelos idealizadores do artefato.

Além disso, Franssen explica que podemos analisar a relação entre usuário, função do artefato e sua finalidade por meio de três instâncias: os tokens (ocorrências), types (tipos) e os kinds (modos). Um token é um exemplar do objeto, por exemplo, "meu celular sobre a mesa", "o carro do meu vizinho", etc. Um kind é um artefato definido por seu papel funcional e seu design capaz de realizar "a função de", por exemplo, servir como um celular, um carro, uma mesa etc. E por type, ele entende um modo mais genético de fabricação, seu histórico de design e construção, identificável por uma quantidade determinada de réplicas (Idem. p.49-50). Por exemplo, um Xbox 360 produzido em 2006 para o mercado da União Europeia. Este type de Xbox possuirá características constitutivas e comportamentais distintivas e tokens limitados.

Neste sentido, há um histórico específico de atribuição de qualidades (e.g. ser um artefato eficiente ou defeituoso) no caso dos tokens em contraste com os types. E o modo como a atribuição é feita em relação ao type é caracterizado como a função herdada mais geral, o que coloca determinado type em uma classe de types. Portanto, contendo uma caracterização mais ampla de funcionalidades. Como critério para se identificar o type de um artefato, Franssen considera o seguinte (Idem. p.50):

'Type X é um bom Type-K' expressa o fato normativo que o token de $\mathrm{X}$ tipicamente tem certas características f e por causa destas características, se uma pessoa $\mathrm{p}$ deseja alcançar o resultado de K-ndo, então $\mathrm{p}$ tem uma razão para usar um token de X para K-ndo ${ }^{4}$.

A expressão K-ndo significa a realização de uma ação no gerúndio (em inglês $K$-ing). Por exemplo, cortando, se deslocando, comunicando, partindo, derretendo, etc.

\footnotetext{
4 'Type $X$ is a good K-type' expresses the normative fact that a token of $X$ typically has certain features $f$ and that because of these features, if a person $p$ wishes to achieve the result of $\mathrm{K}$-ing, then $p$ has a reason to use a token of $X$ for K-ing.
}

\begin{tabular}{|l|c|c|c|c|}
\hline Qevista Dialectus & Ano 9 & n. 19 & Agosto - Dezembro 2020 & p. $162-175$ \\
\hline
\end{tabular}


Note que o fato normativo é determinado por razões de uso. E o mau funcionamento é definido como o token $\mathrm{x}$ caindo sobre o Type-K, mas incapaz de executar a atividade de K-ndo para a qual foi atribuída.

Já os critérios de atribuição das propriedades "bom" e "ruim" são maleáveis e dependentes do contexto. Pois, segundo Franssen, é necessário já ter em mente um padrão regular de usuário e design racionalmente concebido e justificado para se julgar tais propriedades acerca dos artefatos (Idem. pp.52-53). Ainda assim, é possível julgar a qualidade funcional de um artefato a partir das razões dos usuários (Idem. p.54):

\footnotetext{
'x é um K funcionando' / ' $\mathrm{x}$ é um bom K' / 'x serve como um bom K' expressa o fato normativo de que $\mathrm{x}$ tem características $\mathrm{f}$ e que por causa destas características, se uma pessoa $\mathrm{p}$, deseja alcançar o resultado de K-ndo, então $\mathrm{p}$ tem uma razão para usar x para K-ndo. ${ }^{5}$
}

Em termos negativos, de mau funcionamento ou ineficiência, as condições são as seguintes (Ibidem): “"x não é e não faz um K' expressa o fato normativo de que $\mathrm{x}$ tem as características f e por causa destas características, uma pessoa $\mathrm{p}$ tem uma razão para não usar x para K-ndo”. A partir daí, são diferenciadas estas duas condições (mau funcionamento e ineficiência) do seguinte modo:

O que 'x é um K com mau funcionamento' e 'x não é [e] nem não faz um bom K' compartilham é, agora, a falta de certa capacidade física, e o que se distingue entre os dois casos são novamente as características históricas (a história do design de $\mathrm{x}$ ) presente na primeira e ausente na última. (Idem. p.55)

A condição negativa como um todo torna mais explicita a passagem do aspecto funcional e pragmático para o aspecto normativo, tendo em vista que isto implica em um impedimento racional no uso do artefato. Mas, qual é a natureza deste impedimento? Para France (Idem, p.55), há uma hierarquia de fatos normativos para os totens de artefato. $\mathrm{O}$ token $\mathrm{X}$ pode ser útil ou inútil para Knob. Se x for útil, então ou x é um kind-K operacional, ou x é um não-K. Se x não é K, ele pode ser um objeto natural (pedra, galho, etc.) ou um artefato desenvolvido para uma ação diferente de K-ndo, mas que pode servir como um $\mathrm{K}$ ou se fazer um $\mathrm{K}$ a partir dele. Se x for inútil, então x é um não-K e não pode fazer um $K$, ou x está com mau funcionamento.

5 ' $\mathrm{x}$ is a working $\mathrm{K}$ ' / ' $\mathrm{x}$ is a good $\mathrm{K}$ ' / ' $\mathrm{x}$ makes a (good) $\mathrm{K}$ ' expresses the normative fact that $\mathrm{x}$ has features $f$ and that because of these features, if a person $p$ wishes to achieve the result of $K$-ing, then $p$ has a reason to use $\mathrm{x}$ for $\mathrm{K}$-ing.

\begin{tabular}{|l|c|c|c|c|}
\hline Qevista Dialectus & Ano 9 & n. 19 & Agosto-Dezembro 2020 & p. $162-175$ \\
\hline
\end{tabular}


É importante agora retomarmos o que vimos anteriormente. Segundo Bunge, há um caráter prático nos produtos tecnológicos. Segundo argumentei, a aplicação destes artefatos (em especial, de caráter científico) por meio da aplicação de nosso know-how, nos permite mais know-that, que por sua vez, se reverte em aumento de know-how, amplificando nossas ações. Franssen, nos mostra que podemos integrar estes objetos empregados em nossos fazeres (doings) a partir de uma compreensão normativa deles. Desta forma, o que gostaria de indagar é: e quando não estamos a empregar tecnologia na ciência e a própria tecnologia se volta para outros fazeres, os não científicos?

Embora em Bunge haja uma cisão entre objetos para fins científicos e não científicos sua separação é feita com critérios epistêmicos e que pressupõe uma diferença fundamental entre know-that e know-how. Separação que abdico de saída. Meu posicionamento é de que não há separação entre estas dimensões, como não há uma separação a priori entre artefatos para finalidade científica e não científica. Tal finalidade é determinada pelo contexto de uso dos usuários e dos engenheiros. A mudança desta perspectiva permite considerarmos uma dimensão mais humana na compreensão dos artefatos tecnológicos.

Para Franssen, posso argumentar que não há uma distinção clara por parte dos usuários entre as razões teoréticas (de design) de um artefato e as razões práticas (a função de realizar alguma atividade $-K$-ndo). Para ele, a justificativa do sujeito pela escolha e uso de um determinado artefato vincula-se mais aos traços socioculturais que os científicos (Idem. p.57). E é neste sentido que as consequências morais ganham relevância nas praticas tecnológicas. As consequências morais fornecem um caráter deôntico no uso e design do artefato em dado contexto: "Quando uma pessoa p diz 'x (não) deve fazer K em circunstancias c', p expressa a opinião de que p está justificado em esperar que x (não) fará K nas circunstancias $c$. ." (Idem. p.57). Nesta passagem, há a caraterização do papel normativo acerca das classes de objetos que devem e que não devem ser usados para realizar as atividades tecnológicas. Este aparato normativo definirá, em última instância, o que consideraremos como objetos tecnológicos enquanto tal e que, por causa disto, farão parte de nossas práticas.

Com esta caracterização, saímos do aspecto estritamente empírico e vinculado apenas ao domínio epistêmico e passamos para uma caracterização prática e normativa, que potencialmente pode ser aplicada em um domínio mais amplo de entidades tecnológicas. Pois, tudo o que vale em termos formais para a amplificação de

\begin{tabular}{|l|c|c|c|c|}
\hline Qevista Dialectus & Ano 9 & n. 19 & Agosto-Dezembro 2020 & p. $162-175$ \\
\hline
\end{tabular}


ações no âmbito científico pode valer para nossas práticas cotidianas e regulares. Se com $\mathrm{x}$ posso fazer $\mathrm{B}$, mais e melhor, que digamos com y, e fazer B com x está associado com valores positivos (funcionais e racionais), no uso de x posso adicionalmente fazer $\mathrm{C}$. Nestes termos, a tecnologia nos permite fazer mais. Portanto, nosso know-how se vê amplificado por meio de aparatos: conversar a distância, saber e reagir sobre eventos do outro lado do mundo em tempo real, controlar economias com gestos da mão, fazer cirurgias microscópicas, curar doenças com origem molecular, etc. Mas, como compreender a estrutura da amplificação de nosso know-how?

Esta compreensão não será feita com recurso direto ao domínio do empírico. Tomarei a rota da filosofia da linguagem por meio da tese de que tudo que é antes concebível é linguisticamente articulável. Esta escolha facilitará a conexão entre a ação, a dimensão normativa e nossa capacidade de articulação em vista das novas ações que se abrem com o emprego da tecnologia.

A partir desta postura, faço passagem para o domínio específico do inferencialismo linguístico-pragmático que ocorrerá após realizarmos um ajuste metafísico nos pressupostos ontológicos de Franssen e a devida interpretação inferencialista do poder e dever de sua teoria normativa dos artefatos tecnológicos.

\section{Os objetos tecnológicos no quadro referencial de Brandom}

É possível entender os objetos criados pela tecnologia em uma perspectiva inferencial pragmática? Penso que sim. Vimos no decurso da apresentação de Franssen que há fatos normativos: "Cada fato observacional é uma razão, para qualquer pessoa $\mathrm{p}$ aprendendo sobre esse fato, por acreditar em um numero de proposições." ${ }^{6}$ (Idem. p.56). Para Brandom, entretanto, não há fatos como componentes ontológicos últimos. Mas, a normatividade característica dos artefatos tecnológicos pode ser incorporada à sua filosofia.

Contudo, ajustes são necessários. Não há tematizada nas obras Making It explicit (BRANDOM, 1998.) e Articulating Reasons (BRANDOM, 2000.) uma ontologia apoiada em fatos. Para Brandom, as forças motrizes das nossas atividades linguísticas, portando, da cognoscibilidade do real, são as inferências e as asserções que compõem o

6 "Every observational fact is a reason, for any person $\mathrm{p}$ learning of that fact, for believing a number of propositions."

\begin{tabular}{|l|l|l|l|l|}
\hline Q Rovista Dialectus & Ano 9 & n. 19 & Agosto-Dezembro 2020 & p. $162-175$ \\
\hline
\end{tabular}


que ele chama de "jogo de dar e pedir razões". Onde este jogo se constitui um fazer humano por excelência e a dimensão de tematização de todas as nossas outras práticas (BRANDOM, 1998. p.277). Sendo as razões deste jogo os componentes de nossas práticas linguísticas e elementos constitutivos de nossas normas, as regras (OLIVEIRA, 2019. p. 87). Por este motivo, substituirei o conceito fato por razões na definição central de Franssen. Note que esta substituição não afeta a finalidade da teoria normativa dele, apenas modifica seu compromisso ontológico e adiciona maior escopo pragmático. Vejamos o resultado da substituição:

(Franssen-prag.): 'O Type X é um bom Type-K' expressam razões para asserir que o token de $\mathrm{x}$ tipicamente tem certas características $\mathrm{f}$ e por causa destas características, se uma pessoa p deseja alcançar o resultado de K-ndo, então p tem uma razão para usar um token de x para K-ndo.

O jogo de dar e pedir razões não se encerra em uma sequência de asserções e inferências, mas antes, é demarcado pela prática dos participantes, o que Brandom chama de scorekeeping practice (BRANDOM, 1998. pp.185-186). Cada interlocutor acompanha as inferências e asserções de seus pares de modo a manter a consistência com certas relações deônticas. Tais relações são atribuições normativas de autorizações, compromissos e incompatibilidades entre nós e nossas asserções, tais que orientam nossas inferências e, em um sentido amplo, a totalidade de nossas práticas racionais. Uma vez que, é através deste movimento inferencial de asserções carregadas de atribuições deônticas que o conteúdo dos conceitos que usamos é estabelecido. Por exemplo: quando afirmo que Jack é meu gato, estou comprometido com o a asserção de que Jack é um felino. Ao passo que estou autorizado a afirmar que ele tem muitos pelos, ou que tem o péssimo hábito de miar alto à noite. Igualmente, estou impedido de afirmar que ele é um cachorro ou uma ave ou um roedor, etc. Este impedimento é o que Brandom chama de incompatibilidade. São justamente estas relações deônticas com outras asserções que se estabelece o conceito de felino. Em termos mais formais, no decurso de um diálogo, numa interação social, ou até mesmo em uma reflexão, há um conjunto de asserções que podemos fazer acerca do contexto no qual estamos inseridos. Este conjunto é modelado justamente pelos três tipos de relações descritas. A cada asserção que fazemos a modelagem e as ações dos interlocutores orientam nossos próximos passos. Embora as práticas em questão sejam ações eminentemente linguísticas, uma vez que as práticas tecnológicas tenham sido abarcadas pela linguagem, todas as nossas ações desta natureza

\begin{tabular}{|l|c|c|c|c|}
\hline Qevista Dialectus & Ano 9 & n. 19 & Agosto-Dezembro 2020 & p. $162-175$ \\
\hline
\end{tabular}


passam a ser explicitadas e modificadas por nosso jogo de dar e pedir razões, portanto, incorporando as ações tecnológicas.

Diante desta forma de explicar nossas práticas, vejamos agora a leitura inferencialista das ações em razão do emprego de artefatos tecnológicos. Note que as autorizações, compromissos e incompatibilidades podem ser obtidos pela definição de Franssen, substituindo-se razões pelos tipos específicos de relações deônticas que envolvem a prática.

'O Type X é um bom Type-K' expressa o compromisso com a asserção de que o token de x tipicamente tem certas características f e por causa destas características, se uma pessoa p deseja alcançar o resultado de K-ndo, então p tem autorização para usar um token de x para K-ndo.

Note que aqui a passagem do compromisso para autorização implica em uma passagem da expressão linguística para a uma ação não linguística. Entretanto, esta passagem não implica em mudança no pano de fundo linguístico-pragmático da concepção de Brandom: linguagem é um fazer. Neste sentido, a linguagem é um fazer que normatiza, regula e reforma outros fazeres. Portanto, no âmbito das ações, não há mudança de estatuto, tampouco no âmbito de expressão do que se está a fazer. Na dimensão inferencial, também não há alteração. Note que os processos inferenciais característicos de nossa racionalidade estão implícitos na própria passagem do compromisso para a autorização da definição acima. Igualmente, a partir do resultado da autorização, que é a ação de usar um equipamento, podemos retornar rapidamente ao âmbito linguístico, inferindo a partir da ação bem ou malograda de uso do artefato e via a epistemologia confiabilista proposta por Brandom (cf. BRANDOM, 2000. pp.117-122.) uma série de outras autorizações e compromissos.

E a incompatibilidade? Da mesma forma que definimos as outras relações normativas, a incompatibilidade pode ser identificada com a condição negativa de uso de um artefato conforme foi apresentado acima. Assim, podemos fazer a seguinte modificação:

(Franssen-Incompatibilidade): 'x não é e não faz um K' expressa a incompatibilidade de que $\mathrm{x}$ tem as características f e que, por causa dessas características, uma pessoa p está comprometida a não usar x para K-ndo.

Aqui a incompatibilidade tem a forma de um compromisso contrário para p e a negação da autorização para x, pois não está autorizado a K-ndo. Talvez, um modo

\begin{tabular}{|l|c|c|c|c|}
\hline Qevista Dialectus & Ano 9 & n. 19 & Agosto-Dezembro 2020 & p. $162-175$ \\
\hline
\end{tabular}


definitivo de demonstrar o sucesso desta conversão de Franssen para o quadro teórico normativo de Brandom seja a adequação da frase que indica o entrelaçamento normativo e prático:

(Normativo-Brandom):

Quando uma pessoa p diz que: 'x está comprometido a (não) fazer $\mathrm{K}$ em circunstancias c', significa que p expressa a opinião de que ele (p) está autorizado em esperar que x (não) fará $\mathrm{K}$ nas circunstâncias $c$.

Há diversos pontos importantes nesta modificação. O primeiro é a passagem do compromisso para a autorização. O segundo é a incorporação da incompatibilidade às situações de autorização e compromisso. Em terceiro lugar, há aqui e já em Franssen, o caráter dialógico fundamental para o andamento do jogo de dar e pedir razões. A ação de $\mathrm{x}$ desencadeará novas possibilidades para x e para $\mathrm{p}$. Por exemplo, x pode não realizar o que se espera, e neste caso, receber modificações. Ou confirmar seu compromisso em não fazer K, ou mesmo demandar de p razões para justificar o que ele está enunciando.

\section{Conclusão}

Deste modo, é reconhecível dois resultados. O primeiro é que a filosofia de

Brandom é capaz de incorporar a discussão dos artefatos tecnológicos e sua manifestação normativa. De fato, não apenas incorpora como fornece conceitos mais refinados (finegrained). Isto acontece, pois o conceito de razão de Franssen pode ser substituído por autorizações, compromissos e incompatibilidades, que são todos os conceitos componentes das regras do jogo de dar e pedir razões. Assim, abre-se a possibilidade de explorar novos nexos entre estas regulações normativas e, da perspectiva de nosso percurso, habilitando o tratamento mais detalhado do estatuto dos objetos tecnológicos. Visto que a interpretação pragmática que propus ao final da discussão sobre Bunge e o tratamento normativo de Franssen eliminam a distinção a priori entre itens tecnológicos com finalidade científica e não científica, temos que, ao lermos esta discussão a partir da filosofia brandomiana a distinção pode ser retomada com outro estatuto epistemológico, a saber: definimos a finalidade de um item tecnológico por meio de critérios práticos e pragmáticos. Isto que dizer que a definição é dada pelo design do objeto e pela prática racional dos usuários. Note que, por estes não serem condicionantes fixos, é possível definir situações em que a prática dos usuários sobrescreve o design dos engenheiros.

\begin{tabular}{|l|c|c|c|c|}
\hline Qevista Dialectus & Ano 9 & n. 19 & Agosto-Dezembro 2020 & p. $162-175$ \\
\hline
\end{tabular}


Muitos são os casos de situações como estas, há uma longa lista de life hacks que existem disponíveis na internet que servem como exemplo.

Em segundo lugar, os artefatos tecnológicos e sua discussão podem agora ser pensados a partir de um jogo pragmático emergindo de nossas práticas sócio-históricas, neste sentido, os itens tecnológicos fornecem extensões de ações dos agentes, manifestando-se em um contexto normativo, a saber, como entidades acopladas aos indivíduos. Uma intuição captada por Bunge e que pode ser mais bem explorada a partir da filosofia de Brandom. Nestes termos, os objetos operam como extensão de nossas práticas, nossos fazeres (doings). Um objeto por si não desempenha papel ativo em um jogo de dar e pedir razões, mas uma vez que tenha sido incorporado às ações dos agentes, ele abre uma gama de possibilidades de ação, compromissos de uso e efeitos e incompatibilidades. Esta abertura modifica radicalmente o percurso de nossas práticas linguísticas enquanto usuários e enquanto interlocutores. Modificação esta que se estende às próprias regras do jogo. Talvez, mitigar estas modificações seja um passo interessante a se seguir no aprofundamento da interação humano - máquina a partir do quadro conceitual de Brandom.

\section{Referências bibliográficas}

BUNGE, M. Technology as applied Science. In: Technology and Culture. Vol. 7, N. 3, 1966. pp.329-347.

BRANDOM, Robert B. Articulating Reasons: An Introduction to Inferencialism. Cambridge: Harvard University Press, 2000.

Making it Explicit: Reasoning, Representing and Discursive Commitment. Cambridge: Harvard University Press, 1998.

CUPANI, A. Filosofia da Tecnologia: Um Convite. $3^{\text {a } e d . ~ F l o r i a n o ́ p o l i s: ~ E d i t o r a ~ U F S C, ~}$ 2017.

EVANS, R. P. Computer Models of Constitutive Social Practice. In: MÜLLER, V. C. (Ed.) Fundamental Issues of Artificial Intelligence. Oxford: Springer, 2016. [Cap. 23] pp.391-411.

FRANSSEN, M. The Normativity of Artefacts. In: Studies in History and Philosophy of Science Part A. n.37, 2006. pp.42-57.

\begin{tabular}{|l|l|l|l|l|}
\hline Q Povista Dialectus & Ano 9 & n. 19 & Agosto - Dezembro 2020 & p. $162-175$ \\
\hline
\end{tabular}



.; LOKHORST, Gert-Jan; VAN DE POEL, Ibo. Philosophy of Technology. In: The Stanford Encyclopedia of Philosophy. Edward N. Zalta (ed.), 2018. URL = https://plato.stanford.edu/archives/fall2018/entries/technology/ (acesso:29/03/2020).

FUMERTON, R. Epistemologia. Petrópolis: Vozes, 2014.

HECK, R. L. Informação a Partir de Regras: Considerações para a Solução do Escândalo da Dedução pela Semântica Inferencial Pragmática. Fortaleza. 146p. Doutorado, Filosofia. UFC. 2018.

OliveIRA, M. A. de. Ontologia em Debate no Pensamento Contemporâneo. São Paulo: Paulus, 2014.

A Metafísica do Ser Primordial. São Paulo: Loyola, 2019.

SUH, Nam Pyo. Axiomatic Design: Advances and Applications. Oxford/New York: Oxford University Press, 2001.

VERKERK, M. J.; HOOGLAND, J.; STOEP, J. van der. DE VRIES, M. J. Filosofia da Tecnologia: Uma introdução. Viçosa: Ultimato, 2018.

\begin{tabular}{|l|c|c|c|c|}
\hline Q Povista Dialectus & Ano 9 & n. 19 & Agosto - Dezembro 2020 & p. 162-175 \\
\hline
\end{tabular}

